SNUTP $99-022$

\title{
Large Extra Dimension Effects on the Spin Configuration of the Top Quark Pair at $e^{+} e^{-}$Colliders
}

\author{
${ }^{(a)}$ Kang Young Lee, ${ }^{(a, b)}$ H.S. Song, ${ }^{(a)}$ JeongHyeon Song, and ${ }^{(b)}$ Chaehyun Yu. \\ (a) Center for Theoretical Physics, Seoul National University, Seoul 151-742, Korea \\ (b) Department of Physics, Seoul National University, Seoul 151-742, Korea
}

\begin{abstract}
Large extra dimension effects on the spin configuration of the top quark pair at the $e^{+} e^{-} \rightarrow t \bar{t}$ process are studied. It is shown that the $\mathrm{TeV}$ scale quantum gravity effects cause significant deviations from the Standard Model predictions for the spin configuration in the off-diagonal basis: they lead to substantial cross sections of the like-spin states of the top quark pair, which vanish in the SM; they weaken the pure dominance of the processes, the UpDown (Down-Up) spin states for the left-handed (right-handed) beam. In addition it is shown that the angular cut $-0.5<\cos \theta<0$ is very effective to determine the sign of the quantum gravity corrections.
\end{abstract}

Typeset using REVTEX 
There has been an increasing interest in the low scale quantum gravity as an extension of the Kaluza-Klein (KK) scenario. Recently Arkani-Hamed, Dimopoulos, and Dvali (ADD) [1] have suggested that the size of the extra dimensions could be large enough to be detectable if we confine the matter fields to the 4-dimensional world where we live. According to their idea, the weakness of the gravity in our world is caused by the suppression factor from the large extra dimensions since gravitons are the only fields freely propagating in the whole $(4+N)$-dimensional spacetime. Considering the macroscopic Gauss' Law for the Newtonian gravity, ADD have derived the relation between the Planck scale and the size of the extra dimensions as

$$
M_{P l}^{2} \sim M_{S}^{N+2} R^{N},
$$

where $M_{S}$ is the only fundamental scale of nature, of which the value is comparable with the electroweak scale. The $N=1$ case is excluded by this simple relation because the corresponding size of the extra dimension is order of $10^{13} \mathrm{~cm}$ if the $M_{S}$ is at a few $\mathrm{TeV}$ order which is phenomenologically interesting. The $N=2$ case which implies mm scale extra dimensions is not excluded by the current macroscopic measurement of gravitational force [2]. For the cases of $N>2$ there exist no other serious constraints up to now. The hierarchy of the Planck scale $M_{P l}$ and the electroweak scale $M_{W}$ is reduced to the revelation of the effects of the large extra dimensions.

Indeed interesting is that this idea is testable by the collider phenomenologies in the near future. When the graviton momentum does not exceed the scale of $M_{S}$, the spacetime where collisions take place can be approximately described by the linear expansion around the flat metric. Within the framework of the linearized gravity, the effective action in the 4-dimensional spacetime is derived after compactifying the extra dimensions, which leads to corresponding Feynman rules [3, 国. The KK reduction from the whole $(4+N)$-dimension to our 4-dimension yields towers of massive KK states in the 4-dimensional effective theory, of which the massive spectrum is cutoff at the scale $M_{S}$. Each graviton in such KK towers interacts with the ordinary matter fields with the couplings suppressed by the Planck scale. 
The production of a single graviton is enhanced by the kinematic factor and has been studied as a source of the missing energy in the $e^{+} e^{-} \rightarrow \gamma E$ Eor $p \bar{p} \rightarrow \gamma E$ process 4 , effects of massive graviton exchange may be enhanced by the sum of the tower of the KK states and provide various signals in the collider phenomenologies [6 [12]. In particular the spin two nature of the gravitons will result in some characteristic effects on the polarization observables [12].

In the present work, we consider the top quark pair production in the Next $e^{+} e^{-}$Linear Collider (NLC) [13]. It is a promising testing ground for new physics effects because of the heavy mass of top quark. The presence of large extra dimensions affects the top quark pair production at $e^{+} e^{-}$collisions through the interactions between the fermions and the Kaluza-Klein tower of gravitons. In the view point of our 4-dimensional world, the massless gravitons freely propagating in the $(4+N)$-dimensional bulk are massive spin two, spin one and spin zero gravitons under the compactification of the extra dimensional manifold. Since the remarkable successes of the SM in explaining all the high energy experiments can be retained by assuming the $\mathrm{SM}$ fields confined on our world, i.e. $T_{\mu j}=T_{i j}=0$ $(\mu=1, \cdots 4 ; i, j=5, \cdots(4+N))$, only the spin two gravitons and the dilaton modes of the spin zero gravitons interact with the ordinary matter. In addition the coupling of the dilaton modes to the electron is to be neglected at high energy collisions, since it is proportional to the fermion mass. Thus the process $e^{+} e^{-} \rightarrow t \bar{t}$ receives the effects of $\mathrm{TeV}$ scale quantum gravity by the $s$-channel Feynman diagram mediated by the spin two Kaluza-Klein gravitons. Furthermore, the produced top quark is known to be in the unique spin configuration at the polarized $e^{+} e^{-}$collider [14] and the information of the top spin is not lost through hadronization since its lifetime is too short for the top quark to constitute hadrons. We can read out the information on the top polarization through the angular distribution of the decay products [15]. The neutral current nature of the graviton interactions leaves the electroweak decay of top quark pair intact, implying that the SM prediction of the angular correlations between the decay products and the spin orientation of each top quark remains valid [15]. Therefore the spin configuration of the top quark pair can be a probe of the 
effects of KK gravitons.

For the process

$$
e^{-}\left(k_{1}\right)+e^{+}\left(k_{2}\right) \rightarrow t\left(p_{1}\right)+\bar{t}\left(p_{2}\right)
$$

the scattering amplitude of the $s$-channel Feynman diagram mediated by the spin two gravitons summed over the Kaluza-Klein tower can be written by

$$
\begin{aligned}
\mathcal{M}_{G}=\frac{\lambda}{M_{S}^{4}} & {\left[\left(k_{1}-k_{2}\right) \cdot\left(p_{1}-p_{2}\right) \bar{v}\left(k_{2}\right) \gamma_{\mu} u\left(k_{1}\right) \bar{u}\left(p_{1}\right) \gamma^{\mu} v\left(p_{2}\right)\right.} \\
& \left.+\bar{v}\left(k_{2}\right)\left(\not p_{1}-\not p_{2}\right) u\left(k_{1}\right) \bar{u}\left(p_{1}\right)\left(k_{1}-\not k_{2}\right) v\left(p_{2}\right)\right],
\end{aligned}
$$

where the order one parameter $\lambda$ depends on the number of extra dimensions and the compactification models. Since the exact value of the $\lambda$ including sign is not determined unless the full quantum gravity theory is provided, two representative cases of $\lambda= \pm$ are considered in the following discussion [6]. It is also to be noted that the amplitude in Eq.(3) as well as the SM amplitudes at the tree level are CP invariant.

In order to analyze the spin configuration of the top quark pair, let us briefly review a generic spin basis discussed in Ref. [14]. We define the spin states of the top quark and top anti-quark in their own rest-frame by decomposing their spins along the reference axes $\hat{\eta}$ and $\hat{\bar{\eta}}$, respectively. The $\mathrm{CP}$ invariance, which is valid at the tree level even with the large extra dimension effects, does not allow the T odd quantity, i.e. $\vec{\sigma}_{t} \cdot\left(\left.\overrightarrow{k_{1}}\right|_{(t-\text { rest })} \times\left.\overrightarrow{p_{2}}\right|_{(t-\text { rest })}\right)$ where the $\vec{\sigma}_{t}$ is the spin of the top quark, and $\left.\overrightarrow{k_{1}}\right|_{t-\text { rest }}$ and $\left.\overrightarrow{p_{2}}\right|_{t \text {-rest }}$ are the momenta of the electron and the top anti-quark in the rest frame of the top [14,16]. Thus the top and anti-top spins are to lie in the production plane. The spin four-vectors of the top quark pair are chosen to be back-to-back in the zero momentum frame. The $\hat{\eta}$ is expressed by an angle $\xi$, the angle between $\hat{\eta}$ and the top anti-quark momentum in the rest frame of the top quark. The usual helicity basis is obtained by taking $\xi=\pi$.

In this general spin basis the differential cross sections of the $e^{+} e^{-} \rightarrow t \bar{t}$ process with the large extra dimension effects are

$$
\frac{d \sigma}{d \cos \theta}\left(e_{L}^{-} e_{R}^{+} \rightarrow t_{\uparrow} \bar{t}_{\uparrow} \text { or } t_{\downarrow} \bar{t}_{\downarrow}\right)=\frac{N_{c} \pi \alpha^{2} \beta}{2 s}\left|\tilde{A}_{L} \cos \xi-\tilde{B}_{L} \sin \xi\right|^{2},
$$




$$
\begin{aligned}
& \frac{d \sigma}{d \cos \theta}\left(e_{L}^{-} e_{R}^{+} \rightarrow t_{\uparrow} \bar{t}_{\downarrow} \text { or } t_{\downarrow} \bar{t}_{\uparrow}\right)=\frac{N_{c} \pi \alpha^{2} \beta}{2 s}\left|\tilde{A}_{L} \sin \xi+\tilde{B}_{L} \cos \xi \pm \tilde{D}_{L}\right|^{2}, \\
& \frac{d \sigma}{d \cos \theta}\left(e_{R}^{-} e_{L}^{+} \rightarrow t_{\uparrow} \bar{t}_{\uparrow} \text { or } t_{\downarrow} \bar{t}_{\downarrow}\right)=\frac{N_{c} \pi \alpha^{2} \beta}{2 s}\left|\tilde{A}_{R} \cos \xi-\tilde{B}_{R} \sin \xi\right|^{2}, \\
& \frac{d \sigma}{d \cos \theta}\left(e_{R}^{-} e_{L}^{+} \rightarrow t_{\uparrow} \bar{t}_{\downarrow} \text { or } t_{\downarrow} \bar{t}_{\uparrow}\right)=\frac{N_{c} \pi \alpha^{2} \beta}{2 s}\left|\tilde{A}_{R} \sin \xi+\tilde{B}_{R} \cos \xi \mp \tilde{D}_{R}\right|^{2},
\end{aligned}
$$

where $t_{\uparrow}\left(t_{\downarrow}\right)$ denotes the top spin along (against) the $\hat{\eta}, N_{c}$ is the number of color, $\alpha$ is the fine structure constant, $\beta=\sqrt{1-4 m_{t}^{2} / s}$ and

$$
\begin{aligned}
\tilde{A}_{L} & =\frac{1}{2}\left(f_{L L}+f_{L R}\right) \sin \theta \sqrt{1-\beta^{2}}-f_{G} \sin 2 \theta \sqrt{1-\beta^{2}}, \\
\tilde{B}_{L} & =\frac{1}{2}\left[f_{L L}(\cos \theta+\beta)+f_{L R}(\cos \theta-\beta)\right]-f_{G} \cos 2 \theta \\
\tilde{D}_{L} & =\frac{1}{2}\left[f_{L L}(1+\beta \cos \theta)+f_{L R}(1-\beta \cos \theta)\right]-f_{G} \cos \theta \\
\tilde{A}_{R} & =\frac{1}{2}\left(f_{R R}+f_{R L}\right) \sin \theta \sqrt{1-\beta^{2}}-f_{G} \sin 2 \theta \sqrt{1-\beta^{2}}, \\
\tilde{B}_{R} & =\frac{1}{2}\left[f_{R R}(\cos \theta+\beta)+f_{R L}(\cos \theta-\beta)\right]-f_{G} \cos 2 \theta, \\
\tilde{D}_{R} & =\frac{1}{2}\left[f_{R R}(1+\beta \cos \theta)+f_{R L}(1-\beta \cos \theta)\right]-f_{G} \cos \theta .
\end{aligned}
$$

The large extra dimension effects are altogether included in the quantity $f_{G}$ defined by

$$
f_{G}=\frac{\beta s^{2}}{4 \alpha} \frac{\lambda}{M_{S}^{4}} .
$$

Here $f_{I J}$ 's $(I, J=L$ or $R)$ are

$$
f_{I J}=Q_{\gamma}(e) Q_{\gamma}(t)+Q_{Z}^{I}(e) Q_{Z}^{J}(t) \frac{1}{\sin ^{2} \theta_{W}} \frac{s}{s-M_{Z}^{2}}
$$

and $\theta$ is the scattering angle of the top quark with respect to the electron beam. The electric charges and couplings to the $Z$ boson of the electron and the top quark are given by

$$
\begin{aligned}
Q_{\gamma}(e) & =-1 & Q_{\gamma}(t) & =\frac{2}{3}, \\
Q_{Z}^{L}(e) & =\frac{2 \sin ^{2} \theta_{W}-1}{2 \cos \theta_{W}}, & Q_{Z}^{R}(e) & =\frac{\sin ^{2} \theta_{W}}{\cos \theta_{W}}, \\
Q_{Z}^{L}(t) & =\frac{3-4 \sin ^{2} \theta_{W}}{6 \cos \theta_{W}}, & Q_{Z}^{R}(t) & =-\frac{2 \sin ^{2} \theta_{W}}{3 \cos \theta_{W}} .
\end{aligned}
$$

A comment on the behaviors of the $f_{I J}$ 's are in order here for they play a central role in the SM background. Numerical results show that the $f_{I J}$ 's are negative quantity and their 
dependence on the beam energy is weak at high energy. For instance, their numerical values at $\sqrt{s}=500 \mathrm{GeV}$ are

$$
f_{L L}=-1.21, \quad f_{L R}=-0.43, \quad f_{R L}=-0.20, \quad f_{R R}=-0.87
$$

indicating $f_{L L}>f_{L R}$ and $f_{R R}>f_{R L}$.

There exist the angles $\xi_{L}$ and $\xi_{R}$ such that the differential cross sections for the $t_{\uparrow} \bar{t}_{\uparrow}$ and $t_{\downarrow} \bar{t}_{\downarrow}$, i.e., like-spin states vanish for the left- and right-handed electron beam, respectively. It is called the "off-diagonal basis", of which the name originated in this feature [14. This is general in any $2 \rightarrow 2$ process if the $\mathrm{CP}$ is conserved and the spin four-vectors of the outgoing particles are back-to-back in the zero momentum frame.

In the SM, the angles $\xi_{L, R}$ are taken to be

$$
\cos \xi_{I}=-\frac{B_{I}}{\sqrt{A_{I}^{2}+B_{I}^{2}}}, \quad \sin \xi_{I}=-\frac{A_{I}}{\sqrt{A_{I}^{2}+B_{I}^{2}}},
$$

where $I=L, R, A_{I}=\left.\tilde{A}_{I}\right|_{f_{G}=0}$, and $B_{I}=\left.\tilde{B}_{I}\right|_{f_{G}=0}$. The differential cross sections in Eq.(4) are reduced to, in this SM off-diagonal basis,

$$
\begin{aligned}
\frac{d \sigma}{d \cos \theta}\left(e_{L}^{-} e_{R}^{+} \rightarrow t_{\uparrow} \bar{t}_{\uparrow} \text { or } t_{\downarrow} \bar{t}_{\downarrow}\right)= & \frac{N_{c} \pi \alpha^{2} \beta}{2 s} f_{G}^{2}\left[\sin \xi_{L} \cos 2 \theta-\cos \xi_{L} \sin 2 \theta \sqrt{1-\beta^{2}}\right]^{2}, \\
\frac{d \sigma}{d \cos \theta}\left(e_{L}^{-} e_{R}^{+} \rightarrow t_{\uparrow} \bar{t}_{\downarrow} \text { or } t_{\downarrow} \bar{t}_{\uparrow}\right)= & \frac{N_{c} \pi \alpha^{2} \beta}{2 s}\left[\sqrt{A_{L}^{2}+B_{L}^{2}} \mp D_{L}\right. \\
& \left.+f_{G}\left(\cos \xi_{L} \cos 2 \theta+\sin \xi_{L} \sin 2 \theta \sqrt{1-\beta^{2}} \pm \cos \theta\right)\right]^{2}, \\
\frac{d \sigma}{d \cos \theta}\left(e_{R}^{-} e_{L}^{+} \rightarrow t_{\uparrow} \bar{t}_{\uparrow} \text { or } t_{\downarrow} \bar{t}_{\downarrow}\right)= & \frac{N_{c} \pi \alpha^{2} \beta}{2 s} f_{G}^{2}\left[\sin \xi_{R} \cos 2 \theta-\cos \xi_{R} \sin 2 \theta \sqrt{1-\beta^{2}}\right]^{2}, \\
\frac{d \sigma}{d \cos \theta}\left(e_{R}^{-} e_{L}^{+} \rightarrow t_{\uparrow} \bar{t}_{\downarrow} \text { or } t_{\downarrow} \bar{t}_{\uparrow}\right)= & \frac{N_{c} \pi \alpha^{2} \beta}{2 s}\left[\sqrt{A_{R}^{2}+B_{R}^{2}} \pm D_{R}\right. \\
& \left.+f_{G}\left(\cos \xi_{R} \cos 2 \theta+\sin \xi_{R} \sin 2 \theta \sqrt{1-\beta^{2}} \mp \cos \theta\right)\right]^{2},
\end{aligned}
$$

where $D_{I}=\left.\tilde{D}_{I}\right|_{f_{G}=0}$.

There are two important characteristic features of the SM background. First, the differential cross sections for the like-spin states of the top quark pair vanish. Or we have chosen the spin configuration in that way. Secondly, the process for $t_{\uparrow} \bar{t}_{\downarrow}\left(t_{\downarrow} \bar{t}_{\uparrow}\right)$ is dominant when the 
left-handed (right-handed) electron beam is used. This can be easily seen in Eq.(11) since the negative quantities $A$ 's, $B$ 's and $D$ 's are the same order of magnitude. At high energy, the degree of this dominance is close to $100 \%$ [17]. This pure dominance of the Up-Down state for the left-handed electron beam and the Down-Up state for the right-handed one is fairly stable by the one-loop QCD corrections where the soft gluon emissions are dominant so that the QCD corrections are factored out. For instance, the $\mathcal{O}\left(\alpha_{s}\right)$ corrections to the ratio of the cross section for the dominant process to the total cross section is just $\sim 0.01 \%$ while those to the total cross section is about $\sim 30 \%$ at $\sqrt{s} \sim 500 \mathrm{GeV}$ 17, 18.

The TeV scale quantum gravity modifies these two features. First the differential cross sections of the like-spin states acquire the quantum gravity effects. Secondly the presence of $f_{G}$ in the differential cross sections of the dominant spin configurations corresponding to the incident beam polarizations pollutes their pure dominance. In Fig. 1 and 2 we plot the differential cross sections with respect to the top quark scattering angle, broken down to the spin configuration of the top quark pair at $\sqrt{s}=500 \mathrm{GeV}$ with the left- and right-handed beams, respectively. The solid lines denote the SM background, and the dotted (dashed) lines include the quantum gravity effects with $\lambda=+1(\lambda=-1)$. The $M_{S}$ is set to be 2.5 TeV. Figures 3 and 4 illustrate the same observables at $\sqrt{s}=1 \mathrm{TeV}$. At $\sqrt{s}=500 \mathrm{GeV}$, the corrections are small over all the spin configurations, and become a little larger in the case of the right-handed electron beam. Figures 3 and 4 show that the quantum gravity corrections increase with the beam energy. In particular, the like-spin states, which are zero in the tree level SM, gain sizable cross sections.

We present the change of the degree of dominance corresponding to the beam polarization due to the large extra dimensions. Table 1 shows the ratios of the cross section for the dominant process to the total cross section at $\sqrt{s}=500 \mathrm{GeV}$ and $\sqrt{s}=1 \mathrm{TeV}$ for $\lambda= \pm 1$, which are expected to be stable by the one-loop QCD corrections. As the beam energy increases enough, the $\lambda=-1$ case gives rise to more deviations from the SM prediction. And it can be easily seen that the use of the high energy right-handed electron beam is more efficient in detecting the corrections. 


\begin{tabular}{|c|cc|ccc|}
\hline & \multicolumn{2}{|c|}{$\sqrt{s}=0.5 \mathrm{TeV}$} & \multicolumn{3}{c|}{$\sqrt{s}=1 \mathrm{TeV}$} \\
\hline & $\mathrm{SM}$ & $\begin{array}{c}\mathrm{SM}+\mathrm{QG} \\
(\lambda=+1)\end{array}$ & $\mathrm{SM}$ & $\mathrm{SM}+\mathrm{QG}$ & $\mathrm{SM}+\mathrm{QG}$ \\
& & $(\lambda=+1)$ & $(\lambda=-1)$ \\
\hline$\sigma\left(e_{L}^{-} e_{R}^{+} \rightarrow t_{\uparrow} \bar{t}_{\downarrow}\right) / \sigma\left(e_{L}^{-} e_{R}^{+} \rightarrow t \bar{t}\right)$ & $99.26 \%$ & $99.30 \%$ & $96.52 \%$ & $86.62 \%$ & $78.52 \%$ \\
$\sigma\left(e_{R}^{-} e_{L}^{+} \rightarrow t_{\downarrow} \bar{t}_{\uparrow}\right) / \sigma\left(e_{R}^{-} e_{L}^{+} \rightarrow t \bar{t}\right)$ & $99.56 \%$ & $99.58 \%$ & $97.93 \%$ & $82.48 \%$ & $74.78 \%$ \\
\hline
\end{tabular}

Table 1. The ratios of the cross section of the dominant process to the total cross section with $M_{S}=2.5 \mathrm{TeV}$.

Finally let us observe that the angular distribution of the cross sections have provided valuable information on the nature of the interactions between gravitons and fermions. According to the sign of the $\lambda$, the quantum gravity corrections act in a different way. In the dominant processes (the Up-Down spin state with the left-handed electron beam and the Down-Up spin state with the right-handed one), the virtual graviton exchanges cause destructive (constructive) interference with the SM diagrams to the backward direction when $\lambda=+1(\lambda=-1)$. To the forward direction, on the contrary, constructive (destructive) interference occurs when $\lambda=+1(\lambda=-1)$. In the next dominant processes (the Down-Up spin state with $e_{L}^{-}$and the Up-Down one with $e_{R}^{-}$), enormous quantum corrections with $\lambda=+1$ to the backward direction are reduced and dispersed. Therefore we suggest that angular cut, e.g., $-0.5<\cos \theta<0$ be very effective to probe the $\mathrm{TeV}$ scale quantum gravity corrections. The usefulness of the angular cut is demonstrated in Table 2 through the ratios between the cross sections for the next-dominant and dominant processes at $\sqrt{s}=1 \mathrm{TeV}$, where $\Delta \sigma \equiv \int_{-0.5}^{0} d \cos \theta(d \sigma / d \cos \theta)$. This observable clearly discriminates the $\lambda=-1$ case from the $\lambda=+1$ case, as its value is different by a few orders of magnitude. 


\begin{tabular}{|l|ccc|}
\hline & $\mathrm{SM}$ & $\begin{array}{c}\mathrm{SM}+\mathrm{QG} \\
(\lambda=+1)\end{array}$ & $\begin{array}{c}\mathrm{SM}+\mathrm{QG} \\
(\lambda=-1)\end{array}$ \\
\hline$\Delta \sigma\left(e_{L}^{-} e_{R}^{+} \rightarrow t_{\downarrow} \bar{t}_{\uparrow}\right) / \Delta \sigma\left(e_{L}^{-} e_{R}^{+} \rightarrow t_{\uparrow} \bar{t}_{\downarrow}\right)$ & 0.14 & 0.035 & 2.15 \\
$\Delta \sigma\left(e_{R}^{-} e_{L}^{+} \rightarrow t_{\uparrow} \bar{t}_{\downarrow}\right) / \Delta \sigma\left(e_{R}^{-} e_{L}^{+} \rightarrow t_{\downarrow} \bar{t}_{\uparrow}\right)$ & 0.083 & 0.034 & 26.92 \\
\hline
\end{tabular}

Table 2. The ratios of the cross sections for the next-dominant processes to those for the dominant ones with the angular cut $-0.5<\cos \theta<0$ at $\sqrt{s}=1 \mathrm{TeV}$.

In conclusion, we have studied the spin configuration of the top quark pair at the process $e^{+} e^{-} \rightarrow t \bar{t}$ with large extra dimensions. The $\mathrm{TeV}$ scale quantum gravity affects the process through the $s$-channel Feynman diagram mediated by the Kaluza-Klein tower of spin two gravitons. The framework of the off-diagonal basis, in which the cross sections of the like-spin states of the top quark pair vanish, leads to the SM result that the left-handed (right-handed) electron beam almost completely prefer the spin configuration of the top and anti-top spins as Up-Down (Down-Up). The presence of large extra dimensions modifies these features significantly at high energies. It yields non-zero cross sections for the like-spin states and relieves the partiality of the beam polarization for a specific spin configuration of the top quark pair. In addition, it is shown that the angular cut $-0.5<\cos \theta<0$ is very effective to determine the sign of the quantum gravity corrections.

\section{ACKNOWLEDGMENTS}

We would like to appreciate valuable discussions with S.Y. Choi. This work is supported by the Korean Science and Engineering Foundation (KOSEF) through the SRC program of the Center for Theoretical Physics (CTP) at Seoul National University. 


\section{REFERENCES}

[1] N. Arkani-Hamed, S. Dimopoulos, and G. Dvali, Phys. Lett. B429 (1998) 263.

[2] See, e.g. J.C. Long, H.W. Chan, and J.C. Price, Nucl. Phys. B539 (1999) 23.

[3] T. Han, J.D. Lykken and R. Zhang, Phys. Rev. D59 (1999) 105006.

[4] G.F. Giudice, R. Rattazzi, and J.D. Wells, Nucl. Phys. B544 (1999) 3.

[5] E.A. Mirabelli, M. Perelstein, and M.E. Peskin, Phys. Rev. Lett. 82 (1999) 2236.

[6] J. L. Hewett, hep-ph/9811356.

[7] T.G. Rizzo, hep-ph/9904380; hep-ph/9903475; hep-ph/9902273; hep-ph/9901209.

[8] P. Mathews, S. Raychaudhuri, and K. Sridhar, Phys. Lett. B450 (1999) 343.

[9] K. Agashe and N.G. Deshpande, hep-ph/9902263.

[10] C. Balazs, H.-J. He, W. W. Repko, and C.-P. Yuan, hep-ph/9904220.

[11] K. Cheung, hep-ph/9904266; hep-ph/9903294.

[12] K.Y. Lee, H.S. Song, and J. Song, hep-ph/9904355.

[13] E. Accomando et. al. Report No. DESY 97-100.

[14] S. Parke and Y. Shadmi, Phys. Lett. B387 (1996) 199.

[15] T. Arens and L.M. Sehgal, Nucl. Phys. B393 (1993) 46; Phys. Rev. D50 (1994) 4372; G. Mahlon and S. Parke, Phys. Rev. D53 (1996) 4886.

[16] G.L. Kane, G.A. Ladinsky, and C.-P. Yuan, Phys. Rev. D45 (1992) 124.

[17] S. Parke, hep-ph/9807573.

[18] J. Kodaira, T. Nasuno, and S. Parke, Phys. Rev. D59 (1999) 014023. 


\section{FIGURES}

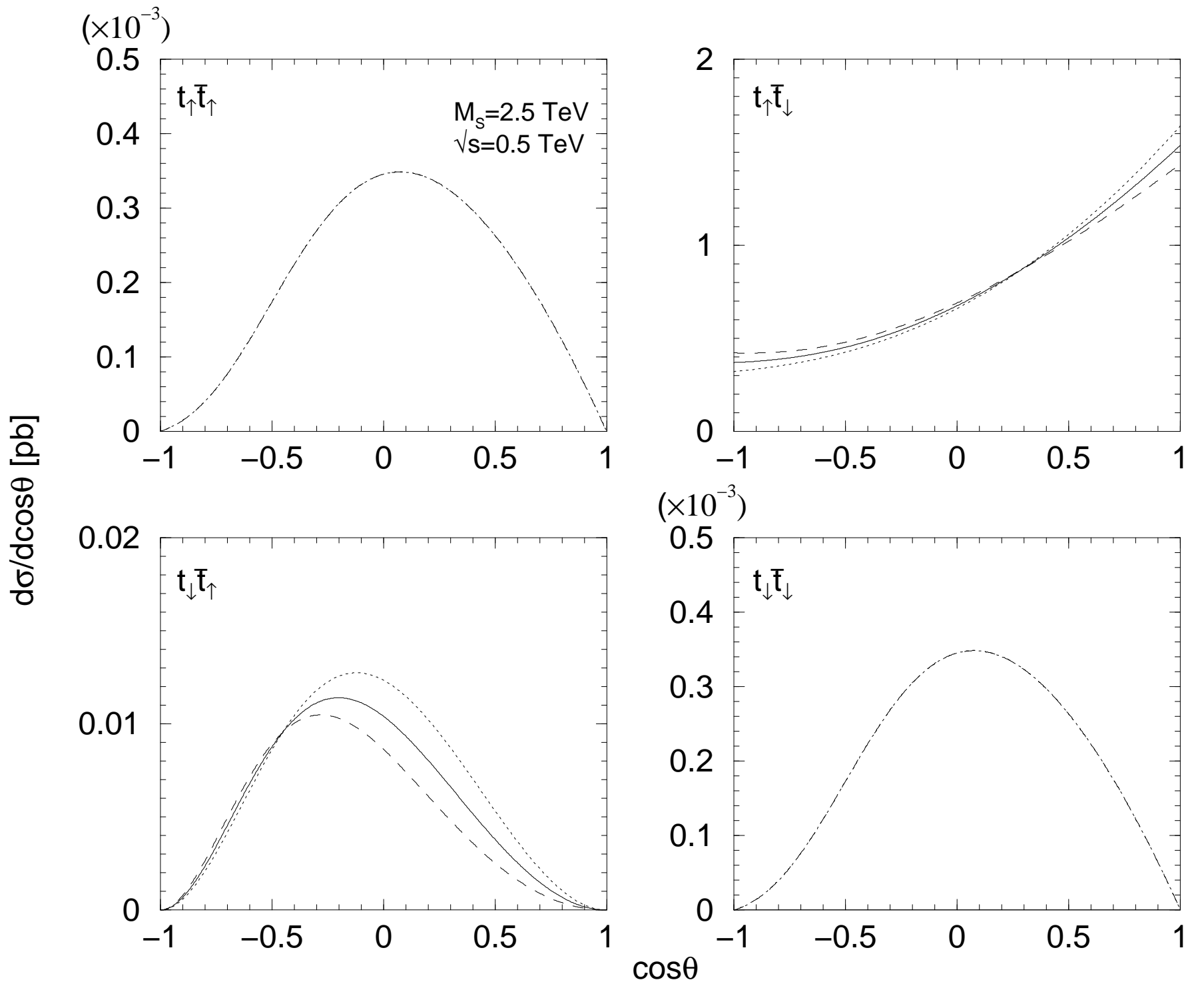

FIG. 1. The differential cross section with respect to the scattering angle of the top quark at $\sqrt{s}=500$ GeV with the left-handed electron beam, broken down to the spin configuration of the top quark pair. The dotted (dashed) line includes the large extra dimension effects when $\lambda=+1$ $(\lambda=-1)$ and $M_{S}=2.5 \mathrm{TeV}$. The solid line denotes the SM background. 

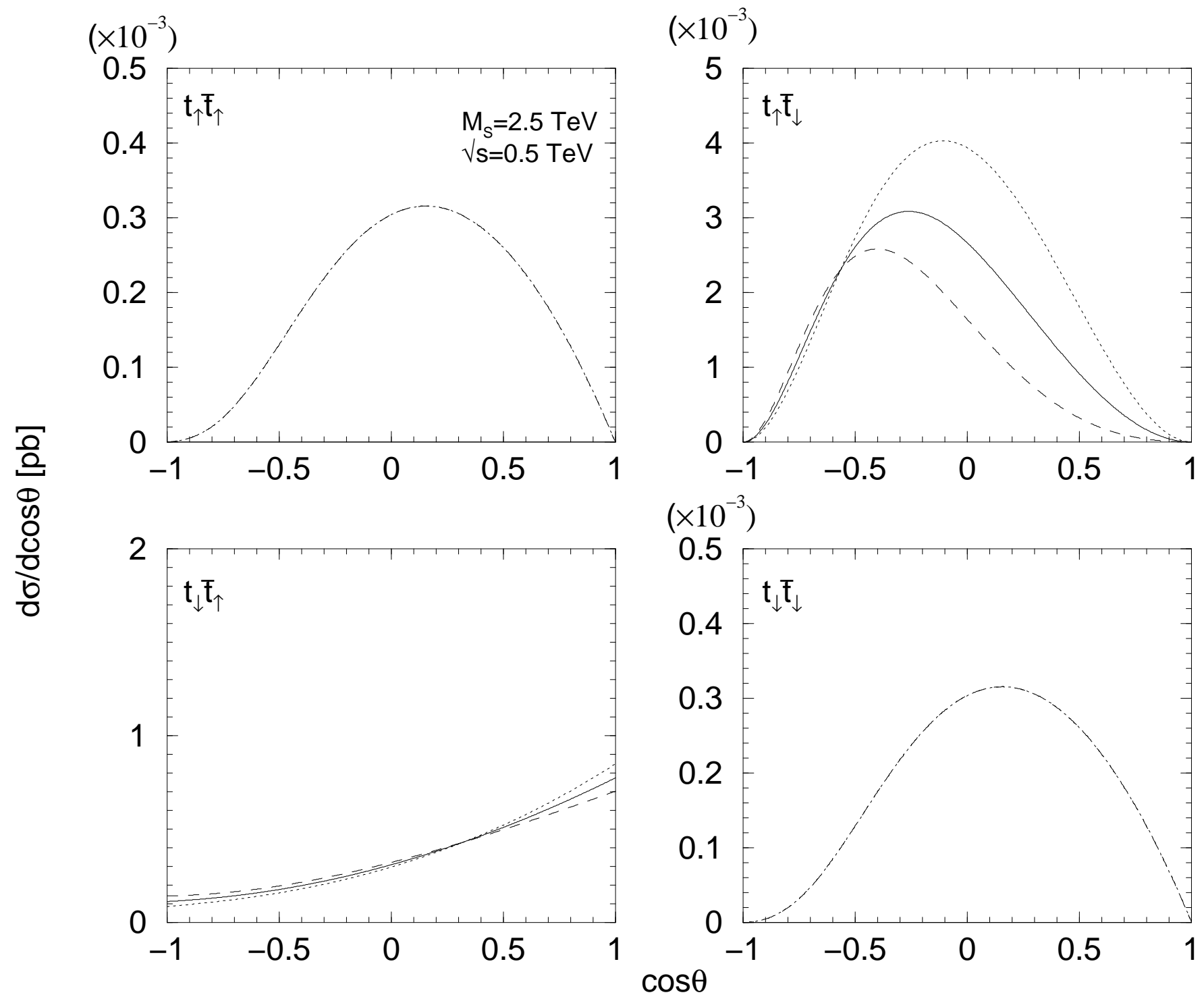

FIG. 2. The differential cross section with respect to the scattering angle of the top quark at $\sqrt{s}=500 \mathrm{GeV}$ with the right-handed electron beam, broken down to the spin configuration of the top quark pair. The dotted (dashed) line includes the large extra dimension effects when $\lambda=+1$ $(\lambda=-1)$ and $M_{S}=2.5 \mathrm{TeV}$. The solid line denotes the SM background. 

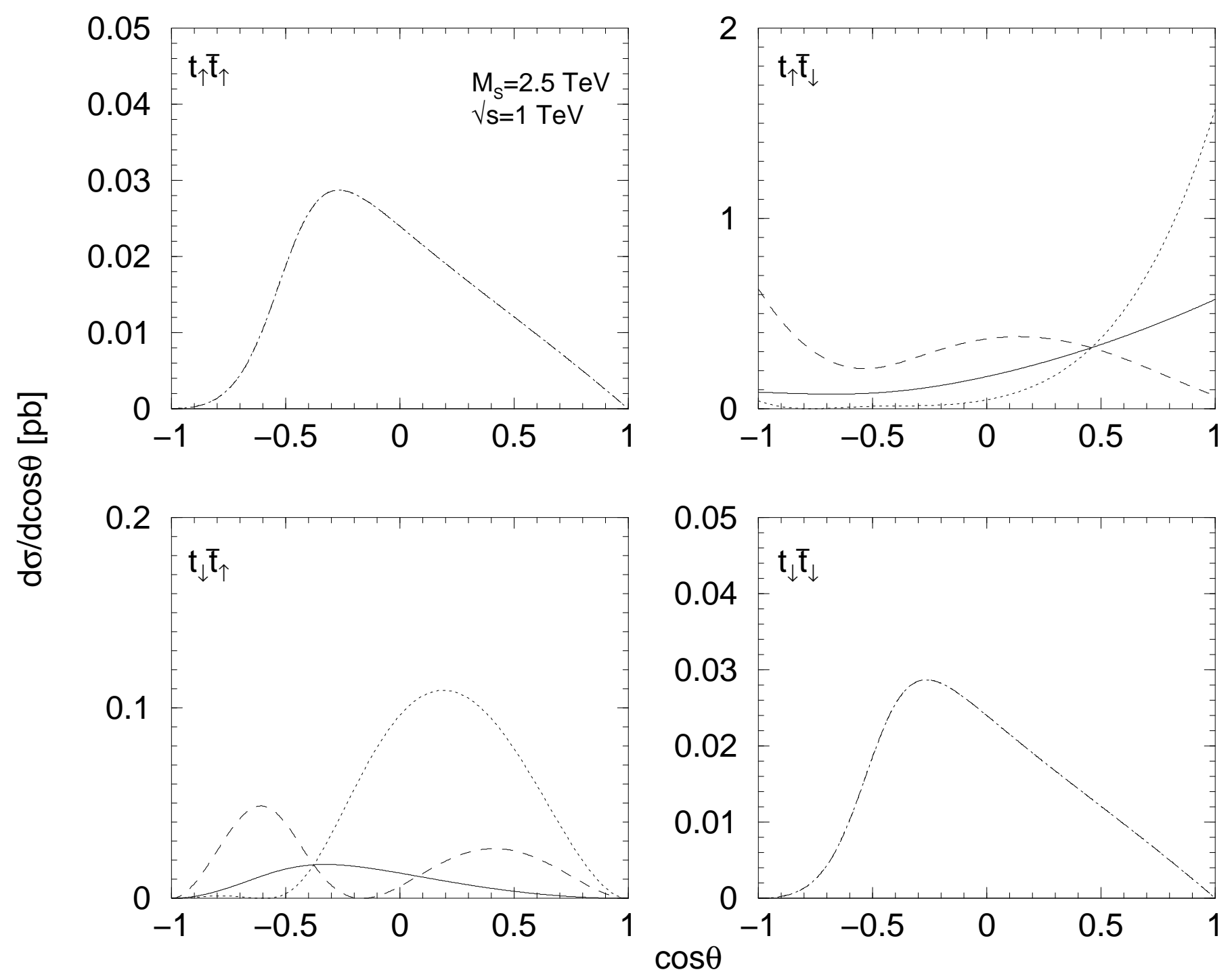

FIG. 3. The differential cross section with respect to the scattering angle of the top quark at $\sqrt{s}=1$ TeV with the left-handed electron beam, broken down to the spin configuration of the top quark pair. The dotted (dashed) line includes the large extra dimension effects when $\lambda=+1$ $(\lambda=-1)$ and $M_{S}=2.5 \mathrm{TeV}$. The solid line denotes the SM background. 

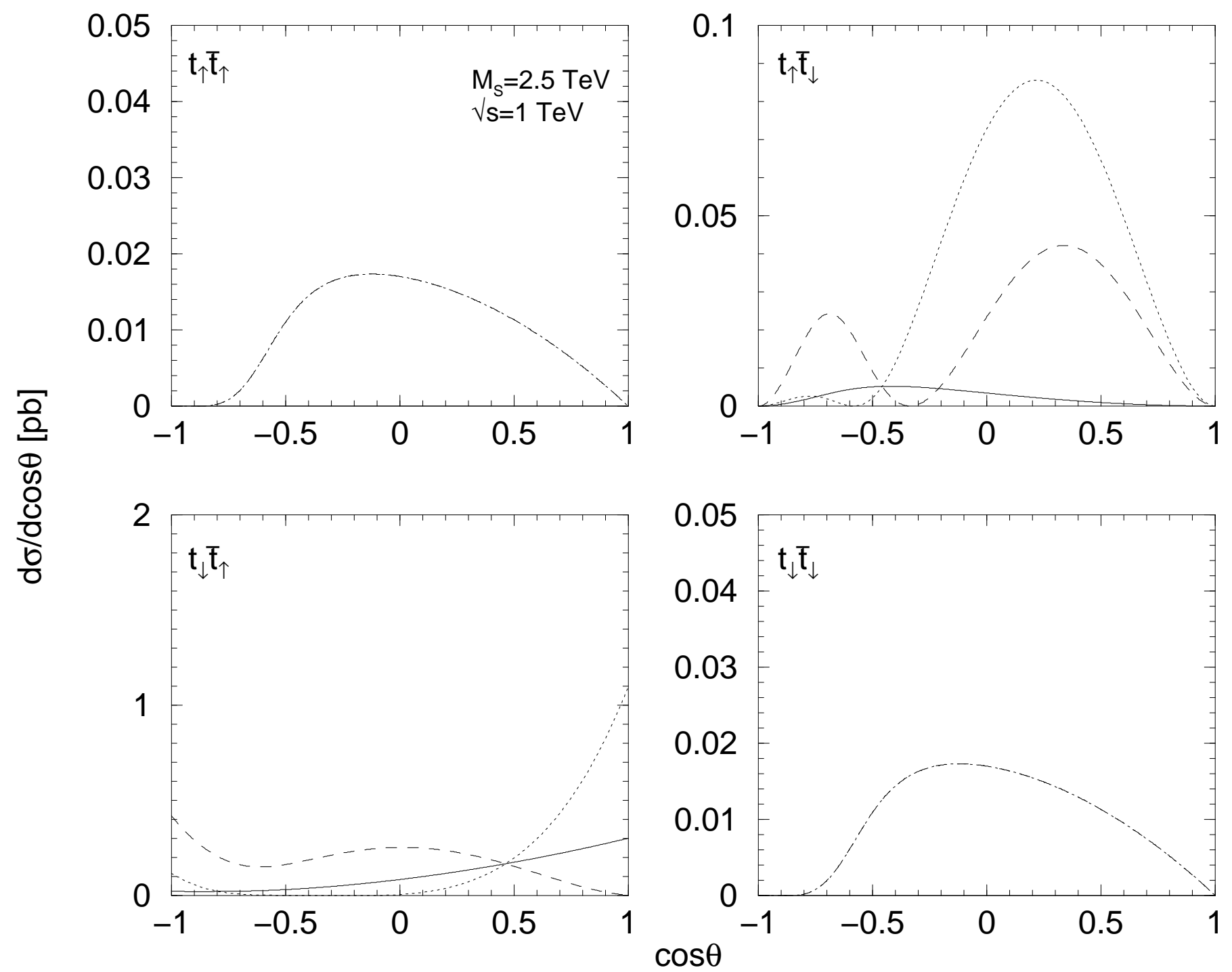

FIG. 4. The differential cross section with respect to the scattering angle of the top quark at $\sqrt{s}=1$ TeV with the right-handed electron beam, broken down to the spin configuration of the top quark pair. The dotted (dashed) line includes the large extra dimension effects when $\lambda=+1$ $(\lambda=-1)$ and $M_{S}=2.5 \mathrm{TeV}$. The solid line denotes the SM background. 